\title{
A new type of square columnar liquid crystalline phases formed by facial amphiphilic triblock molecules
}

\author{
Bin Chen ${ }^{a}$, Ute Baumeister ${ }^{b}$, Siegmar Diele ${ }^{b}$, Malay Kumar Das ${ }^{b}$, Xiangbing Zeng $^{c}$, Goran Ungar $^{c}$ \\ and Carsten Tschierske ${ }^{a}$
}

\begin{abstract}
${ }^{a}$ Martin-Luther-University Halle-Wittenberg, Institute of Organic Chemistry, Kurt-Mothes Str. 2, D-06120 Halle, Germany, e-mail: Tschierske@chemie.uni-halle.de

${ }^{b}$ Martin-Luther-University Halle-Wittenberg, Institute of Physical Chemistry, Mühlpforte 1, D-06108 Halle, Germany

${ }^{c}$ Department of Engineering Materials and Centre for Molecular Materials, University of Sheffield, Robert Hadfield Building Mappin Street, Sheffield S1 3JD, Great Britain
\end{abstract}

\section{Synthesis and analytical data of compounds 2 a-c}

Compounds 2a-c were synthesized from the corresponding carboxylic acids, ${ }^{1}$ by activation as pentafluorophenyl esters, followed by aminolysis with 1-amino-1-deoxy-D-glucitol (D-Glucamin). In a typical procedure (synthesis of 2a) DCC $(157 \mathrm{mg}, 0.76 \mathrm{mmol})$ was added at $0^{\circ} \mathrm{C}$ to a solution of 11-(4,4"-didecyloxy-p-terphenyl-2'-yloxy)-3,6,9-trioxaundecanoic acid (0.52 g, $0.69 \mathrm{mmol})$ dissolved in dry THF $(50 \mathrm{~mL})$. After $30 \mathrm{~min}$, the reaction mixture was allowed to warm up to r.t., then pentafluorophenol (141 $\mathrm{mg}, 0.77 \mathrm{mmol})$ was added, and the reaction mixture was stirred at r.t. for $24 \mathrm{~h}$. 1-Amino-1-deoxy-D-glucitol $(0.63 \mathrm{~g}, 3.5 \mathrm{mmol})$ was added and the resulting mixture was stirred for another $24 \mathrm{~h}$. Afterwards, the precipitates were filtered off and the washed with $\mathrm{CHCl}_{3}$ $(5 \times 5 \mathrm{~mL})$, the combined solutions were evaporated in vacuum, the residue was purified by column chromatography on silica gel with $\mathrm{CHCl}_{3} / \mathrm{MeOH}$ as eluent and repeated crystallized from EtOAc/n-hexane.

2a: ${ }^{1} \mathrm{H}-\mathrm{NMR}\left(\mathrm{CDCl}_{3}, J / \mathrm{Hz}, 400 \mathrm{MHz}\right) \delta=7.57$ (bs, $\left.1 \mathrm{H}, \mathrm{NH}\right), 7.51-7.46$ (m, $\left.4 \mathrm{H}, \mathrm{Ar}-\mathrm{H}\right), 7.30(\mathrm{~d}$, $\left.{ }^{3} J(\mathrm{H}, \mathrm{H})=7.9,1 \mathrm{H}, \mathrm{Ar}-\mathrm{H}\right), 7.17\left(\mathrm{dd},{ }^{3} J(\mathrm{H}, \mathrm{H})=7.9,{ }^{4} J(\mathrm{H}, \mathrm{H})=1.5,1 \mathrm{H}, \mathrm{Ar}-\mathrm{H}\right), 7.11(\mathrm{~s}, 1 \mathrm{H}, \mathrm{Ar}-\mathrm{H})$, 6.94-6.87 (m, $4 \mathrm{H}, \mathrm{Ar}-\mathrm{H}), 3.2-4.2\left(\mathrm{~m}, 26 \mathrm{H}, \mathrm{OCH}, \mathrm{OCH}_{2}\right), 1.83-1.77$ (m, $\left.4 \mathrm{H}, \mathrm{CH}_{2}\right), 1.49-1.41$ (m, $\left.4 \mathrm{H}, \mathrm{CH}_{2}\right), 1.26\left(\mathrm{bs}, 24 \mathrm{H}, \mathrm{CH}_{2}\right), 0.87\left(\mathrm{t},{ }^{3} \mathrm{~J}(\mathrm{H}, \mathrm{H})=6.5,6 \mathrm{H}, \mathrm{CH}_{3}\right) ;{ }^{13} \mathrm{C}-\mathrm{NMR}\left(\mathrm{CDCl}_{3}, 100 \mathrm{MHz}\right) \delta$ $=171.8,158.9,158.2,155.8,140.9,133.1,130.9,130.5(2 \mathrm{C}), 130.4,129.1,127.9(2 \mathrm{C}), 119.8$, 114.8 (2C), $114.0(2 \mathrm{C}), 111.7,73.0,72.6,72.0,71.0,70.7,70.5,70.2,70.1(2 \mathrm{C}), 69.7,68.6,68.2$, 68.1, 63.9, 42.2, 31.9 (2C), 29.6 (2C), 29.6 (2C), 29.5, 29.5, 29.4, 29.4, 29.3 (2C), 26.1, 26.1, 22.7 (2C), $14.1(2 \mathrm{C}) ; \mathrm{C}_{52} \mathrm{H}_{81} \mathrm{NO}_{12} \cdot \mathrm{H}_{2} \mathrm{O}$ (Cal.) $\mathrm{C}: 67.14 \%$, H: $8.99 \%$, N: $1.50 \%$, (Found) C: $67.04 \%$, H: $9.06 \%, \mathrm{~N}: 1.42 \%$.

2b: ${ }^{1} \mathrm{H}-\mathrm{NMR}\left(\mathrm{CDCl}_{3}, J / \mathrm{Hz}, 400 \mathrm{MHz}\right) \delta=7.61\left(\mathrm{bt},{ }^{3} J(\mathrm{H}, \mathrm{H})=5.6,1 \mathrm{H}, \mathrm{NH}\right), 7.51-7.48(\mathrm{~m}, 4 \mathrm{H}$, Ar-H), $7.31\left(\mathrm{~d},{ }^{3} J(\mathrm{H}, \mathrm{H})=7.9,1 \mathrm{H}, \mathrm{Ar}-\mathrm{H}\right), 7.17\left(\mathrm{~d},{ }^{3} J(\mathrm{H}, \mathrm{H})=7.9,1 \mathrm{H}, \mathrm{Ar}-\mathrm{H}\right), 7.11(\mathrm{~s}, 1 \mathrm{H}, \mathrm{Ar}-\mathrm{H})$, 6.95-6.88 (m, $4 \mathrm{H}, \mathrm{Ar}-\mathrm{H}), 4.46$ (bs, $1 \mathrm{H}, \mathrm{OH}), 3.3-4.2\left(\mathrm{~m}, 34 \mathrm{H}, \mathrm{OCH}, \mathrm{OCH}_{2}, \mathrm{OH}\right), 1.81-1.74(\mathrm{~m}$, $\left.4 \mathrm{H}, \mathrm{CH}_{2}\right), 1.46-1.41\left(\mathrm{~m}, 4 \mathrm{H}, \mathrm{CH}_{2}\right), 1.20-1.39\left(\mathrm{~m}, 24 \mathrm{H}, \mathrm{CH}_{2}\right), 0.87\left(\mathrm{t},{ }^{3} \mathrm{~J}(\mathrm{H}, \mathrm{H})=6.5,6 \mathrm{H}, \mathrm{CH}_{3}\right)$; ${ }^{13} \mathrm{C}-\mathrm{NMR}\left(\mathrm{CDCl}_{3}, 100 \mathrm{MHz}\right) \delta=171.7,158.8,158.1,155.9,140.9,133.1,130.8,130.5(2 \mathrm{C})$, 130.4, 129.1, 127.9 (2C), 119.7, 114.8 (2C), 114.0 (2C), 111.6, 73.0, 72.5, 71.9, 70.9, 70.9, 70.4, 70.4, 70.3, 70.2, 70.1 (2C), 69.7, 68.5, 68.2, 68.1, 63.9, 42.1, 31.9 (3C), 29.6 (2C), 29.6 (2C), 29.5, 29.4, 29.4, 29.3 (2C), 26.1, 26.1, 22.7 (2C), $14.1(2 \mathrm{C}) ; \mathrm{C}_{54} \mathrm{H}_{85} \mathrm{NO}_{13} \cdot \mathrm{H}_{2} \mathrm{O}$ (Cal.) C: $66.57 \%$, H: $9.00 \%$, N: $1.44 \%$, (Found) C: $66.74 \%$, H: $9.03 \%$, N: $1.31 \%$. 
2c: ${ }^{1} \mathrm{H}-\mathrm{NMR}\left(\mathrm{CDCl}_{3}, J / \mathrm{Hz}, 400 \mathrm{MHz}\right) \delta=7.68\left(\mathrm{t},{ }^{3} J(\mathrm{H}, \mathrm{H})=6.0,1 \mathrm{H}, \mathrm{NH}\right), 7.52-7.48(\mathrm{~m}, 4 \mathrm{H}, \mathrm{Ar}-$ $\mathrm{H}), 7.32\left(\mathrm{~d},{ }^{3} J(\mathrm{H}, \mathrm{H})=7.9,1 \mathrm{H}, \mathrm{Ar}-\mathrm{H}\right), 7.18\left(\mathrm{dd},{ }^{3} J(\mathrm{H}, \mathrm{H})=7.9,{ }^{4} J(\mathrm{H}, \mathrm{H})=1.7,1 \mathrm{H}, \mathrm{Ar}-\mathrm{H}\right), 7.12(\mathrm{~d}$, $\left.{ }^{4} J(\mathrm{H}, \mathrm{H})=1.7,1 \mathrm{H}, \mathrm{Ar}-\mathrm{H}\right), 6.96-6.88(\mathrm{~m}, 4 \mathrm{H}, \mathrm{Ar}-\mathrm{H}), 4.42\left(\mathrm{~d},{ }^{3} J(\mathrm{H}, \mathrm{H})=5.6,1 \mathrm{H}, \mathrm{OH}\right), 4.15(\mathrm{t}$, $\left.{ }^{3} J(\mathrm{H}, \mathrm{H})=4.7,2 \mathrm{H}, \mathrm{OCH}_{2}\right), 3.3-4.05\left(\mathrm{~m}, 40 \mathrm{H}, \mathrm{OCH}_{2}, \mathrm{OCH}, \mathrm{OH}\right), 1.83-1.77\left(\mathrm{~m}, 4 \mathrm{H}, \mathrm{CH}_{2}\right), 1.49-$ $1.41\left(\mathrm{~m}, 4 \mathrm{H}, \mathrm{CH}_{2}\right), 1.39-1.20\left(\mathrm{~m}, 24 \mathrm{H}, \mathrm{CH}_{2}\right), 0.89-0.85\left(\mathrm{t},{ }^{3} \mathrm{~J}(\mathrm{H}, \mathrm{H})=6.5,6 \mathrm{H} \mathrm{CH}_{3}\right) ;{ }^{13} \mathrm{C}-\mathrm{NMR}$ $\left(\mathrm{CDCl}_{3}, 100 \mathrm{MHz}\right) \delta=171.7,158.8,158.1,155.9,140.9,133.1,130.8,130.5(2 \mathrm{C}), 130.4,129.1$, 127.9 (2C), 119.6, $114.8(2 \mathrm{C}), 114.0(2 \mathrm{C}), 111.7,73.0,72.6,72.0,71.0,70.8,70.5,70.5,70.4,70.4$ (2C), 70.3, 70.2, 70.1, 69.7, 68.4, 68.2 (2C), 68.1 (2C), 63.9, 42.2, 31.9 (2C), 29.6 (2C), 29.6 (2C), 29.5, 29.4 (2C), 29.4, 29.3 (2C), 26.1, 26.122 .7 (2C), $14.1(2 \mathrm{C}) ; \mathrm{C}_{58} \mathrm{H}_{93} \mathrm{NO}_{15} \cdot \mathrm{H}_{2} \mathrm{O}$ (Cal.) C: 65.57 \%, H: $9.01 \%$, N: $1.32 \%$, (Found) C: $65.41 \%, \mathrm{H}: 9.02 \%, \mathrm{~N}: 1.22 \%$.

\section{Electron density reconstruction for the $\mathrm{Col}_{\text {squ }}$ phase of compound $\mathbf{2 b}$}

Synchrotron small-angle diffraction experiments were performed on Station 16.1 at the SRS at Daresbury, U.K. Powder samples in capillaries were held in a modified Linkam temperaturecontrolled stage. A double focused highly collimated monochromatic beam was used, with a Daresbury quadrant multiwire detector. Sample to detector distance was 1.3 metres.

Table 1. Index, $d$-spacings ( $d_{\text {obs }}$ : experimental; $d_{\text {calc }}$ :calculated), intensities and phases of reflections used to reconstruct electron densities of the $\mathrm{Col}_{\text {squ }}$ phase in compound $\mathbf{2 b}$.

\begin{tabular}{|c|c|c|c|c|}
\hline$h k$ & $d_{\text {obs }}(\AA)$ & $d_{\text {calc }}(\AA)$ & $I(h k)$ & $\phi(h k)$ \\
\hline 10 & 40.8 & 40.8 & 100 & $\pi$ \\
\hline 11 & 28.8 & 28.8 & 4.04 & $\pi$ \\
\hline 20 & 20.4 & 20.4 & 5.64 & $\pi$ \\
\hline 21 & 18.2 & 18.2 & 0.16 & $\pi$ \\
\hline 22 & 14.3 & 14.4 & 0.12 & $\pi$ \\
\hline 30 & 13.5 & 13.6 & 0.21 & 0 \\
\hline 31 & 12.8 & 12.9 & 0.06 & 0 \\
\hline
\end{tabular}

The space group of the structure is $p 4 m m$, with unit cell parameters $a=40.8 \AA$. The diffraction intensities $I(h k)$ were measured from the powder diffraction pattern recorded at $80^{\circ} \mathrm{C}$, after cooling the sample from the isotropic phase. The intensities are Lorentz and multiplicity corrected. Fourier reconstruction of the electron density is carried out using the general formula:

$\rho(x y)=\Sigma_{h k} F(h k) \exp [2 \pi i(h x+k y)]=\Sigma_{h k} \sqrt{I(h k)} \exp [2 \pi i(h x+k y)+i \phi(h k)]$.

Here $\phi(h k)$ is the phase of the $(h k)$ reflection.

\footnotetext{
${ }^{1}$ The synthesis of these compounds will be reported in a separate paper.
} 